\title{
Pulsed $\mathrm{Nd}^{3+}$ :YAG Laser Assisted Micro-dimple Formation in Chromium Films under Different Ambient Conditions
}

\author{
V. Ezhilmaran ${ }^{1}$, L. Vijayaraghavan ${ }^{1}$, N. J. Vasa ${ }^{2}$, S. Ganesan ${ }^{3}$, N. K. Cherian ${ }^{3}$ \\ ${ }^{1}$ Department of Mechanical Engineering, Indian Institute of Technology Madras, Chennai-600036, INDIA \\ E-mail:me13d062@smail.iitm.ac.in \\ ${ }^{2}$ Department of Engineering Design, Indian Institute of Technology Madras, Chennai-600036, INDIA \\ ${ }^{3}$ India Pistons Ltd, Chennai-600011, INDIA
}

\begin{abstract}
In this paper, a pulsed laser-assisted micro-dimple formation on chromium film deposited on a cast iron piston ring under different ambient conditions, such as deionized water, coaxially flowing Ar inert gas $(200 \mathrm{kPa})$, atmospheric air and vacuum $(0.003 \mathrm{~Pa})$ is reported. A pulsed nanosecond $\mathrm{Nd}^{3+}$ : YAG laser with a wavelength of $532 \mathrm{~nm}$, pulsed repetition rate of $10 \mathrm{~Hz}$ and with a pulse width of 6 ns was used. The key laser variables selected for study is pulse energy $(5-30 \mathrm{~mJ})$ and number of pulses $(50,100$ pulses). The geometrical features like diameter, depth and aspect ratio which are the important attributes that influence the quality of a drilled micro-dimples are comprehensively investigated. In all the ambient conditions, the diameter and the depth of microdimples increased with increase in the laser ablation energy. On the other hand, the diameter increased and the depth of micro-dimple decreased as the ambient condition changed from vacuum, air, inert gas and water. In the case of water ambience, the overall efficiency of laser ablation was lower than that of other media. Nevertheless, it offered process advantage in terms of less heat affected zone and micro cracks.
\end{abstract}

DOI: $10.2961 / \mathrm{jlmn} .2016 .02 .0007$

Key words: laser drilling, piston ring, micro-dimple, pulsed laser texturing, recast layer.

\section{Introduction}

There is an increasing interest in laser assisted micro manufacturing application for automotive industry, such as micro-dimple formation on piston rings, cylinder liners and bearings, due to improvement in the engine tribology. For example, in the case of piston rings, pulsed laser ablation technique can be used to ablate the material to produce micro grooves on the surface of the piston rings. These grooves store oil and thereby reduce the friction between the piston rings and cylinder liner segments. The reduced friction wear increases the life of the piston rings. Laser assisted micro-dimple formation and texturing of piston ring have been studied by different research groups $[1,2]$. Laser assisted texturing has various advantages, such as flexibility in control of texture size, can be used for treating wider range of materials with less heat affected zone. In particular, $\mathrm{Nd}^{3+}$ :YAG laser being operated in a pulsed mode can also be considered for such texturing application because of large peak power and short pulse width resulting in narrow heat affected zone. It also offers shorter wavelength which enables better beam absorption by metallic materials and generates less plasma in laser drilling applications [3]. The material removal mechanism involved in laser beam machining is by intense heating causes melting and vaporization of the material where the beam is focused resulting in a dimple formation [4]. Owing to the high intensity of the laser beam pulse, surface area adjacent to the micro-dimple also undergoes melting and rapid re-solidification. This collateral damage around the laser irradiated zone is unavoidable when using a high power laser surface processing, leading to an increased roughness and change in micro structural properties around the micro-dimples [5].
The laser parameters, such as average power, pulse energy, pulse duration, pulse frequency, focal position etc., can possibly provide a means for minimizing the above defects [6]. The defects were also be reduced by changing the environment conditions [7]. When laser pulses irradiate the material in air environment, the plasma cloud generated over the ablated portion causes a plasma shielding effect. At high pulse repetition rates, the shielding effect may be even more prominent due the plasma cloud formation. Thus, the effective material removal does not take place [8]. All of these plasma-induced effects degrade beam quality, resulting in poor feature quality and repeatability for laser materials processing. In addition, the laser energy that can be delivered to the target is reduced, which results in a lower efficiency for materials processing. Also plasma shielding limits the repetition rate of the high speed lasers as well as the processing speed [9]. Hence to improve the metal removal and quality, the materials should be ablated in various other environments, such as vacuum and coaxially assisted inert gas. In a vacuum chamber, the plasma-cloud expands faster. Hence the material removal was improved. It also helps to utilize the high repetition rate [10]. By using assist gas as environment, absorptive vapours formed during vaporization can be removed and the laser pulse can freely ablate the material [11]. In the case of nano-second, pulsed laser, the formation of debris around the ablated portion was more prominent. Hence the material was also irradiated by placing it inside water to study the reduction in heat affected zone and recast layer and to investigate the quality of the micro-dimples [12].

In this work, a nano-second pulsed laser is used to ablate chromium deposited piston ring in different ambient conditions using a solid-state laser assisted technique. This 
work attempts to analyze the size and depth of the microdimple by changing various laser parameters like pulse energy and number of pulses. The micro-dimple quality is investigated with the presence of recast layer, heat affected zone and micro cracks in different ambient conditions, such as air, water, inert gas (Ar) and vacuum.

\section{Experimental details}

The experimental set up is shown in Fig. 1 and various ambient conditions for laser ablation are shown in Fig. 2. A Q-switched, pulsed $\mathrm{Nd}^{3+}$ : YAG laser with a wavelength of $532 \mathrm{~nm}$, a pulsed duration of $6 \mathrm{~ns}$ (full width at half maximum) and a pulse repetition rate of $10 \mathrm{~Hz}$ was used. Various ambient conditions, such as still deionised water (water level $2 \mathrm{~mm}$ above work piece), coaxially flowing Argon inert gas $(200 \mathrm{kPa})$, atmospheric air and vacuum $\left(3 \times 10^{-3} \mathrm{~Pa}\right)$ were considered for the study. The pulse energy from 5 to $30 \mathrm{~mJ}$ during four series of shots $(5,8,18,30 \mathrm{~mJ})$ for 50 and 100 number of pulses were used to produce micro-dimples on the surface of molychrome ceramic film ( $99.5 \%$ chromium, $0.4 \%$ molybdenum, $0.1 \%$ cobalt). The film with a thickness of $150 \mu \mathrm{m}$ was deposited on a piston ring made with spheroidal graphite iron. The laser beam was focused down to a spot size approximately $14 \mu \mathrm{m}$ in diameter using a quartz lens with a focal length of $100 \mathrm{~mm}$. The depth of focus (Rayleigh length) was estimated to be around $160 \mu \mathrm{m}$. The beam was focused on the top of the surface. The depth of focus combined by the focusing positioning accuracy allowed consistent dimple formation.

The entry side micro-dimple diameter was measured by using the images obtained by a scanning electron microscope (FEI, Quanta 200). The depth of the microdimples formed was recorded by using a confocal microscope (Olympus, LEXT OLS 4000 model). An aspect ratio, $\varepsilon$, which was defined as a ratio of depth to diameter

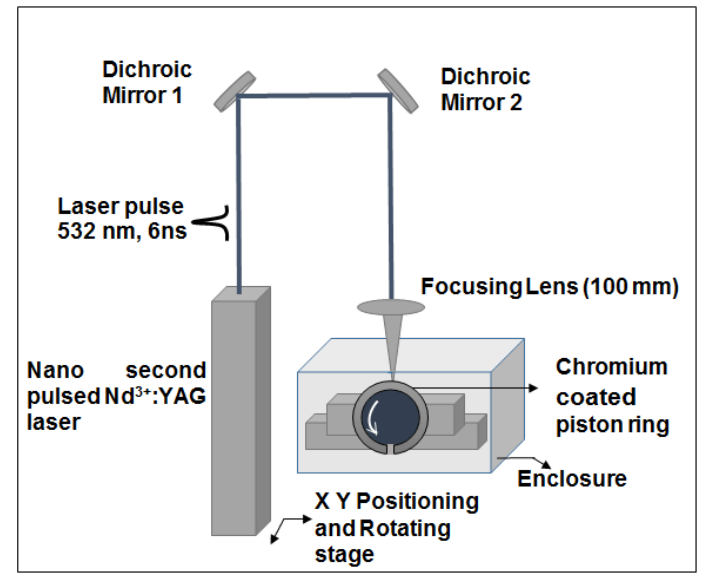

Fig. 1 Experimental set up.

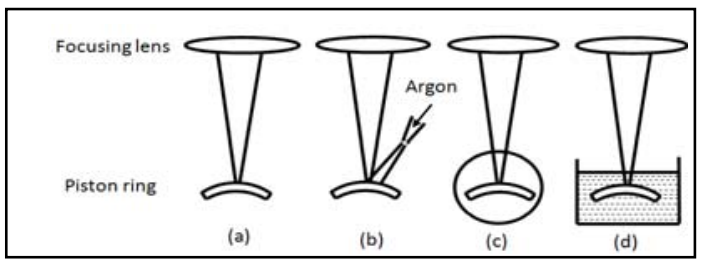

Fig. 2 Different ambient environment for laser ablation: a) Air, b) Coaxially assisted Ar inert gas, c) Vacuum and d) Water. of a dimple, was analyzed. The surface morphology in terms of recast layer, heat affected zone and the microcracks around the dimples were also studied. Before measuring hardness the surface around the dimple was cleaned by diamond polishing to prevent any disturbances by oxide layer that was formed around the micro-dimple during laser irradiation while measuring hardness. The hardness was measured by a micro Vickers hardness tester (Future tech, FM-707). An indentation load of $100 \mathrm{gm}$ for a period of 10 seconds using a narrow rhombus shaped diamond indenter was used.

\section{Results and discussion}

\subsection{Influence of pulse energy and number of pulses on diameter and depth}

The effect of increasing pulse energy on the diameter as well as depth of the micro-dimple was studied for different pulse energy values ranging from 5 to $30 \mathrm{~mJ}$ for 50 and 100 number of pulses. Various pulse energies used are 5, 8, 18, $30 \mathrm{~mJ}$ for 50 and 100 number of pulses. Figs. 3 and 4 shows diameter of dimple formation with respect to different pulse energy in different ambient conditions for 50 and 100 pulses, respectively.

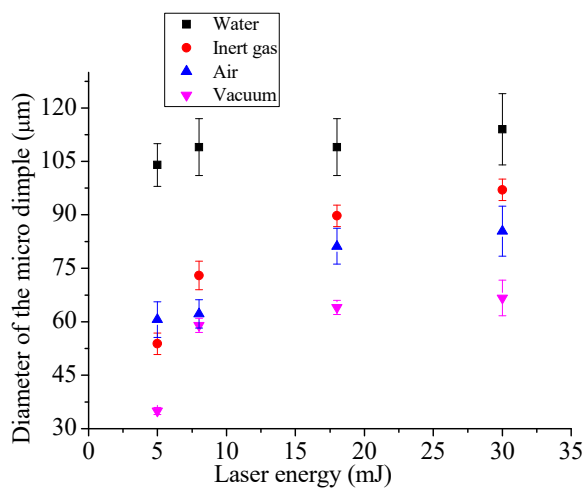

Fig. 3 Laser energy versus diameter of micro-dimple for different ambient conditions (Energy 5, 8, 18, $30 \mathrm{~mJ}$, No of pulses=50).

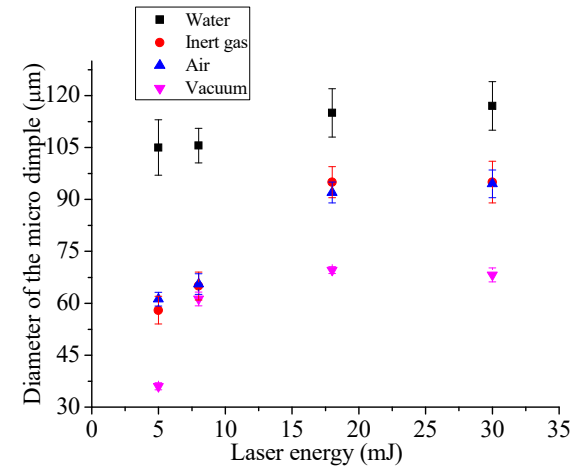

Fig. 4 Laser energy versus diameter of micro-dimple for different ambient conditions (Energy 5, 8, 18, $30 \mathrm{~mJ}$, No of pulses=100).

The measurements of diameters of micro-dimples were performed by taking average of inscribed and circumscribed circles drawn over the micro-dimple. The graphs indicate that an increase in the energy and number of pulses of the incident laser pulse yields larger size dimples, but the rate of increase in diameter reduces with increase in number of pulses. As the laser percussion drilling is based on removing material by a sequence of pulses, each pulse removes a certain volume of material, so that the entire sequence of pulses can achieve deep micro- 
dimple with diameters ranging from $104 \mu \mathrm{m}$ to $117 \mu \mathrm{m}$ for water medium, $61 \mu \mathrm{m}$ to $95 \mu \mathrm{m}$ for air, $54 \mu \mathrm{m}$ to $95 \mu \mathrm{m}$ for inert gas (Ar), $35 \mu \mathrm{m}$ to $68 \mu \mathrm{m}$ for vacuum from both 50 and 100 pulses. The results shows that the diameter obtained in water is larger followed by inert gas and air, while in vacuum it is the smallest.

Based on Figs. 3 and 4, it is clear that with increase in number of pulses, the dimple diameter increases but to a limited extent. This is attributed to the melt expulsion after subsequent pulses has to come out through the entry side of the micro-dimple making the diameter of the micro-dimple bigger. But the rate at which the diameter of the microdimple increases is more for higher energy $(18,30 \mathrm{~mJ})$. Figure 5 shows typical SEM images of laser micro-dimple at $5 \mathrm{~mJ}$ and $30 \mathrm{~mJ}$ energy with 100 pulses at different ambient conditions. Figure 5(a) shows that, in air the splatter was significant and the size of splatter was larger as compared to other ambient conditions. On the other hand, minimum splatter was observed during laser ablation in water.

The influence of energy and number of pulses on depth of the dimple is shown in Figs. 6 and 7. The depth of dimple obtained in vacuum condition was ranging from 32 $\mu \mathrm{m}(5 \mathrm{~mJ}$ and 50 pulses $)$ to $76 \mu \mathrm{m}$ ( $30 \mathrm{~mJ}$ and 100 pulses $)$. Although higher depths were obtained while using inert gas when compared to air ranging from $30 \mu \mathrm{m}(5 \mathrm{~mJ}$ and 50 pulses) to $69 \mu \mathrm{m}$ ( $30 \mathrm{~mJ}$ and 100 pulses), the presence of dross inside the dimple made it difficult to measure the exact depth of the dimple in inert gas environment in which the depth varies from $24 \mu \mathrm{m}$ ( $5 \mathrm{~mJ}$ and 50 pulses) to $46 \mu \mathrm{m}$ (30 $\mathrm{mJ}$ and 100 pulses). While in water the depth was minimum $6 \mu \mathrm{m}(5 \mathrm{~mJ}$ and 50 pulses $)$ to $29 \mu \mathrm{m}(30 \mathrm{~mJ}$ and 100 pulses) due to various energy losses like scattering inside water and reflection loss from the surface of water.

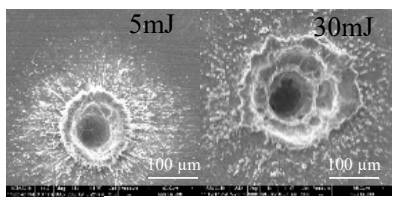

(a)

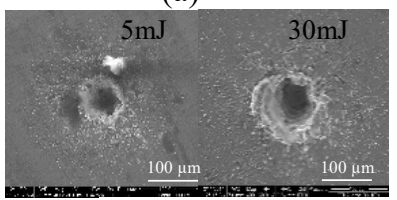

(c)

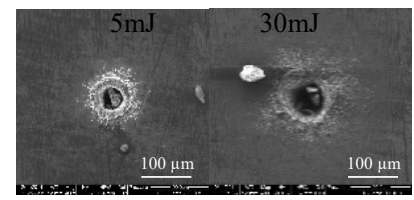

(b)

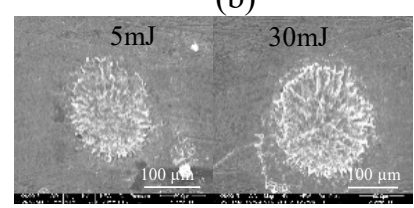

(d)
Fig. 5 Variation of micro-dimple dimension for 100 pulses (a) Air, (b) Vacuum, (c) Inert gas (Ar) and (d) Water.

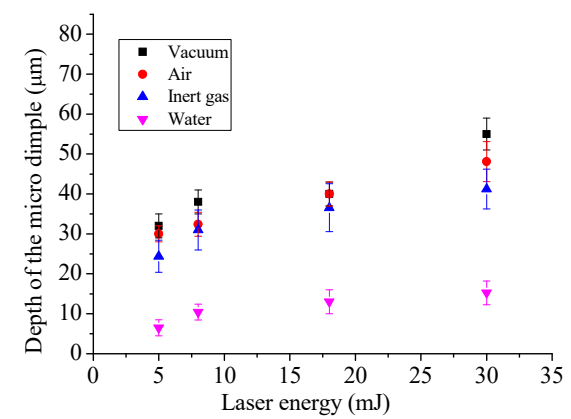

Fig. 6 Laser energy versus depth of micro-dimple for different ambient conditions (Energy 5, 8, 18, $30 \mathrm{~mJ}$, No of pulses=50).

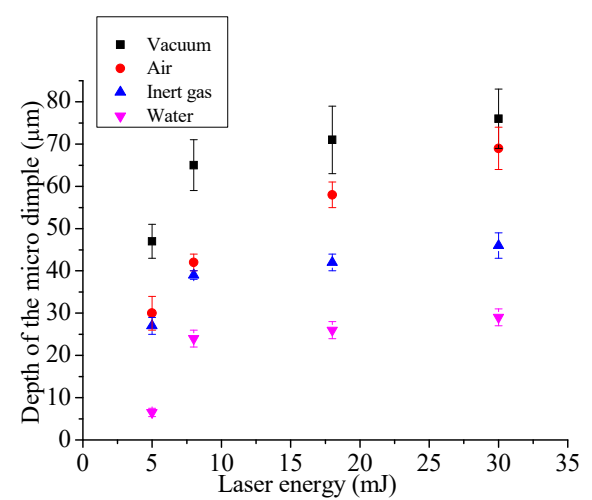

Fig. 7 Laser energy versus depth of micro-dimple for different ambient conditions (Energy 5, 8, 18, $30 \mathrm{~mJ}$, No of pulses=100).

\subsection{Aspect ratio}

Though the diameter of the dimple increased initially with the increase of energy, after a certain input energy, the increase in the dimple diameter was less. On the other hand, the depth of dimple increased with increase in number of pulses. Among the various ambient conditions used in this study, high aspect ratio was attained in vacuum with $\varepsilon=1.30$ followed by air with $\varepsilon=0.73$, with inert gas $\varepsilon=0.60$ and with water $\varepsilon=0.24$ at 100 pulses. The aspect ratio, $\varepsilon$, was increased with the increase in the number of pulses as compared to that when increase in the laser energy. The influence of increase in number of pulses was more as compared to the increase in the laser energy to attain higher aspect ratio. The aspect ratio can be controlled by selecting appropriate laser processing parameters and ambient media.

\subsection{Analysis of recast layer formation}

During irradiation, a recast layer is formed on the inside walls of the micro-dimple. This layer is formed by resolidification of vaporised or molten material on the walls of the micro-dimple, and it is in general not evenly distributed along the micro-dimple circumference and depth. The recast layer influences the accuracy, repeatability of the micro-dimple size and geometry. It is also expected to influence heat conduction properties and increased heat affected zone (HAZ) width and thermal stresses, which can lead to the formation of cracks. The occurrence of coarse recast is particularly associated with melt expulsion during percussion drilling.

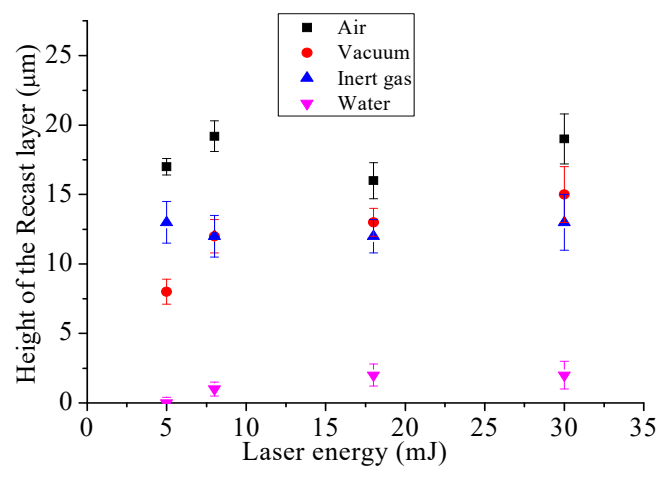

Fig. 8 Height of the recast layer bulges as a function of laser energy for different ambient conditions, No of pulses $=50$. 


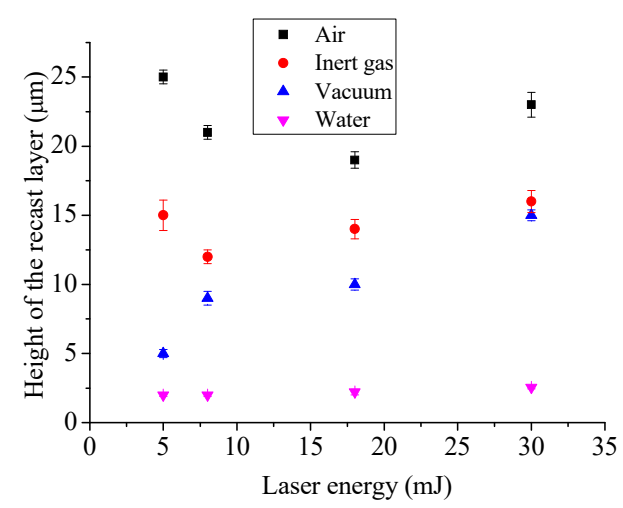

Fig. 9 Height of the recast layer around the micro-dimple as a function of laser energy for different ambient conditions, No of pulses $=100$.

Based on Figs. 8 and 9, the recast layer height around the micro-dimples was found to be higher during ablation in air followed by vacuum, inert gas and water as an ambient medium. Figure 10 shows confocal microscopy profile of the recast layer formed under different ambient conditions. The height of the recast layer was different corresponding to the laser ablation energy under different ambient conditions. The thin and high recast layer formed during ablation in air as shown in Fig. 10(a) was difficult to remove during subsequent cleaning operation, like diamond polishing. In case of inert gas as the medium, the pressure of the gas was not sufficient to remove the projections while using less energy and less number of pulses. Also the dross formation was clearly seen in and around the dimple as shown in Fig. 10(b) and it could be removed easily by diamond polishing. In vacuum, particularly at high energy the recast layer was minimized due to vaporization driven metal removal. The presence of large droplets in the vicinity and within the micro-dimple indicated that melt was ejected energetically away from the micro-dimple in the form of lumps as shown in Fig.10(c). Comparatively, small recast layer was observed in the case of underwater laser ablation. Almost all the melt expulsion in molten state was expected to be transported away by water. This was verified by the absence of large droplets in the vicinity of the micro-dimple as shown in Fig.10 (d). (a)

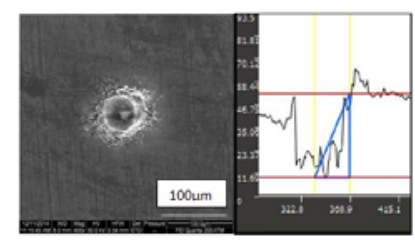

(c)

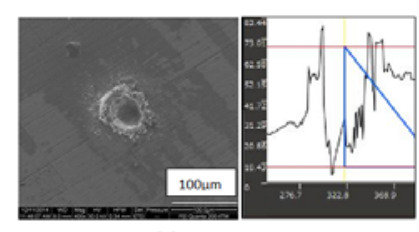

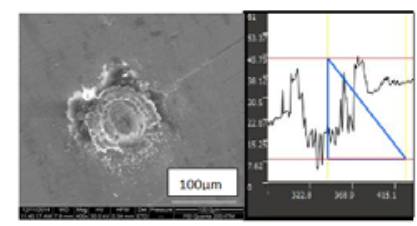

(b)

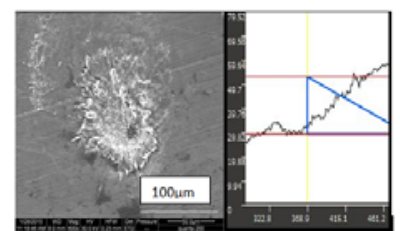

(d)
Fig. 10 SEM images and the corresponding depth profile of the micro-dimple made in (a) Air, (b) Inert gas (Ar), (c) Vacuum and (d) Water. All micro-dimples were formed with $5 \mathrm{~mJ}$ and 50 pulses.
To minimise the recast layer height, parameters should be chosen that allow the material to be expelled from the micro-dimple effectively. This in general means short pulses at high intensity, which changes the material removal mechanism from molten ejection to evaporation dominated process. The use of a suitable process gas and liquid medium may be beneficial to some extent.

\subsection{Analysis of Micro cracks}

Micro cracks result from excessive thermal stresses, and are more likely to occur when a thick recast layer is present. Micro cracks also extended well into the parent material. In air and inert gas medium the crack formation was observed in and around the ablated material with high energy pulses $(18 \mathrm{~mJ}$ and $30 \mathrm{~mJ})$. In vacuum cracks are observed even in less energy. Fig. 11 shows typical microphotograph of the recast layer and thin film where formation of crack was observed with the pulse energy of $30 \mathrm{~mJ}$ (50 pulses) in air ambience. It was mostly profound when using high intensity pulse. At high intensity, the material was expected to be heated enormously resulting in high thermal stresses around the laser ablation zone. Use of low intensity pulse and short pulse duration are the means of minimising the likelihood of micro-cracks. Fewer cracks were found in water medium having improved cooling capacity.

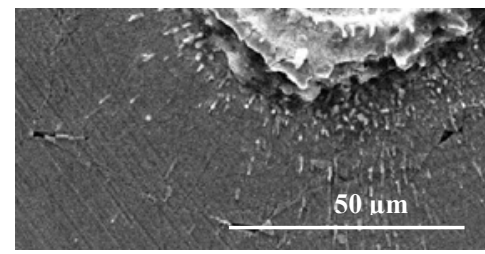

Fig. 11 Formation of cracks in the material ablated in air $(30 \mathrm{~mJ}$ and 50 pulses).

\subsection{Analysis of heat affected zone (HAZ)}

Heat affected zone (HAZ) surrounding the laser assisted micro-dimple formation was determined where the film was not melted during the laser treatment, however mechanical property, such as micro-hardness was altered. This alteration can be harmful causing stresses that reduce the strength of the base material, leading to material failures. Fig. 12 shows a typical heat affected zone around the dimple formation in Ar inert gas medium with $8 \mathrm{~mJ}$ input energy and 50 pulses.

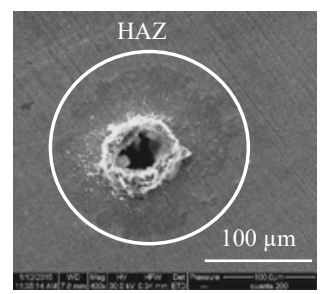

Fig. 12 Heat affected zone around the micro-dimple made in inert gas environment ( $8 \mathrm{~mJ}$ and 100 pulses).

The extent of heat affected zone was obtained by measuring change is hardness values along the circumference of the micro-dimple. Before taking hardness the dimple is carefully polished by diamond paste with 
particle size of $3 \mu \mathrm{m}$ and $1 \mu \mathrm{m}$. The resulting smooth surface around the micro-dimple is shown in Fig. 13.

The hardness value around the micro-dimple obtained at $5 \mathrm{~mJ}$ and with 50 pulses in each environment is measured by using micro Vickers hardness test and the obtained values are shown in Fig. 14. Micro Vickers hardness measurements were performed from the microdimple circumference. In the case of a sample treated in vacuum ambience, the harness value was measured as 1056 $\mathrm{HV}$ at $15 \mu \mathrm{m}$ from the circumference of the dimple. It reduced to around $940 \mathrm{HV}$ at $80 \mu \mathrm{m}$ from the circumference of the micro-dimple. This was matching with the film hardness before the laser treatment. While in air and inert gas the highest hardness value attained is 963 $\mathrm{HV}$ and $975 \mathrm{HV}$ at $15 \mu \mathrm{m}$ near the circumference of the dimple and it reduced the hardness value of the film before the laser treatment at $60 \mu \mathrm{m}$ from the circumference of the dimple. In case of laser treatment in water, no significant change in hardness value was found around the dimple.
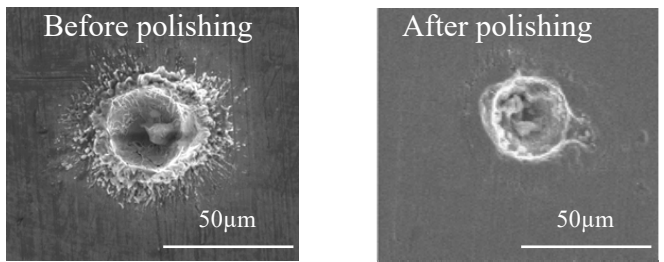

Fig. 13 Diamond polishing of micro-dimple made in vacuum (5mJ and 50 pulses).

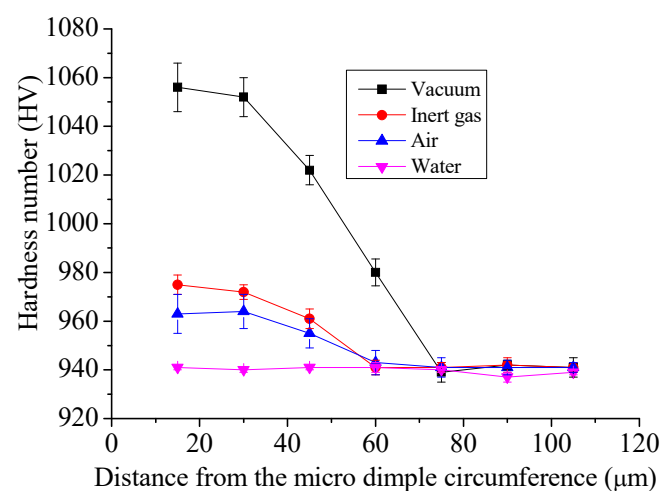

Fig. 14 Measurement of Vickers hardness as a function of radial distance from the micro-dimple circumference for different ambient conditions (Energy $5 \mathrm{~mJ}$ and 50 pulses).

\subsection{Influence of ambient conditions in micro-dimple characteristics}

The main phenomena in removal of the material in air ambience is vaporization and melts expulsion induced by recoil pressure of plasma and vapour formed during irradiation [13]. In air ambience as melting dominated vaporization, the melt expulsion was more, and it resulted in thin recast layer. The increase in dimple diameter with the increase in the input energy and number of pulses was also attributed to the increase in the recoil pressure resulting in increase in the melt expulsion. Hence further polishing was needed to eliminate the effect of hardness due to oxide formation. Although there is no distinct difference in diameter and depth of the micro-dimple obtained in inert gas ambience when compared to air ambience, the height of the recast layer was minimized. This was attributed to the pressure of the inert gas jet force, which might have pushed the melt radially outward from the micro-dimple. At the same time low-assisted gas pressure was unable to remove the excess heat generated at the micro-drilling zone as well as unable to assist the removal of ejected material. This phenomenon causes high heat affected zone.

Though very narrow and deeper dimples were obtained in vacuum compared to other ambience, such as air, inert gas and water, heat affected zone was measured to be larger. In vacuum, particularly at high energy the recast layer was minimized due to vaporization driven metal removal [10]. The melt expulsion which is the main reason for the increase of entry side diameter of the micro-dimple was expected to be less in vacuum ambience. Hence the diameter of the micro dimple was smaller as compared to that in air.

On the other hand, decrease in depth and increase in diameter of dimples in water ambience is attributed to the factors, such as plasma formation and water waves created by vaporization of water. These factors during the underwater laser ablation are expected to impede the laser energy to reach the material surface which ultimately reduces the material removal [14]. Also the increase in diameter of the micro-dimple ablated under water is due to scattering of laser energy in air-water interface. The underwater laser ablation processes has relatively low overall efficiency compared to air, gas and vacuum assisted laser ablation process due to various reasons like high convective loss in water from the hot melt layer, reflection loss from the surface of the water and scattering loss by the water vapour, which ultimately reduce the transparency of the liquid. It offers advantages like less HAZ and less micro cracks. The projection like structure seen inside the spot is due to the formation of oxide layer which can be easily removed by diamond polishing.

\section{Conclusion}

A Q-switched, ns-pulsed $\mathrm{Nd}^{3+}$ : YAG laser was used to study the dimple formation on automotive piston ring. The dimple quality, in terms of both geometrical features and surface morphology was significantly influenced by the pulse energy, number of pulses and ambient condition, such as vacuum, inert gas, air and water. In all the ambient conditions, with increase in laser irradiation energy, diameter and depth of micro-dimples also increased. However the size of diameter increased and the depth of micro-dimple decreased as the ambient condition changed from vacuum, air, inert gas (Ar) and water. The laser focusing condition and confinement of the laser produced plasma is expected to influence the diameter and depth of the micro-dimple formation. Among the various ambient conditions used in this study, high aspect ratio of around $\varepsilon=1.3$ was attained in vacuum and a low aspect ratio of around $\varepsilon=0.24$ was attained in water. Appropriate aspect ratio can be attained by selecting appropriate laser processing parameters, such as number of laser pulses, energy, and ambient media.

In terms of surface morphology, recast layer around the micro-dimples was formed and the height was ranging from 16 to $25 \mu \mathrm{m}$ in air, 5 to $15 \mu \mathrm{m}$ in vacuum, 13 to 15 
$\mu \mathrm{m}$ in inert gas and 0 to $2 \mu \mathrm{m}$ in water ambience. Surface cracks on films treated under vacuum, inert gas and air with high pulse energy were observed near dimple formation. On the other hand, no surface cracks were formed in water ambience. The presence of HAZ was measured by micro Vickers hardness test. The hardness value near the micro-dimple circumference was very high in vacuum followed by inert gas, air and water. In the case of water ambience, the overall efficiency of laser ablation is lower than that of air, inert gas and vacuum ambience. Nevertheless, it offers process advantage in terms of less HAZ and less micro cracks.

\section{Acknowledgements}

The support for the work from the SEM and MEMS laboratories in IIT Madras is gratefully acknowledged. The authors also wish to thank R.Sriram, M.Lavanya, and Srinagalakshmi Nammi for their help in experimentation and characterization of the samples.

\section{Reference}

[1] Ravi Bathe, V. Sai Krishna, S. K. Nikumb, and G. Padmanabham: Appl. Phys., A117, (2014) 117.

[2] G. Ryk and I. Etsion: Wear, 261, (2006) 792.

[3] S. Bandyopadhyay, JK Sarin Sundar, G. Sundararajan, and S. V. Joshi: J. Mater. Process. Technol., 127, (2002) 83.

[4] J. Meijer, K. Du, A. Gillner, D. Hoffmann, V. S. Kovalenko, T. Masuzawa, A. Ostendorf, R. Poprawe, and W. Schulz: CIRP Ann. Manuf. Technol., 51, (2002) 531.

[5] Mishra, Sanjay, and Vinod Yadava: Opt. Laser Technol., 48, (2013) 461.

[6] R. Biswas, A. S. Kuar, S. Sarkar, and S. Mitra: Opt. Laser Technol., 42, (2010) 23.

[7] J. Sun, and J. P. Longtin: J. Appl. Phys., 89, (2001) 8219.

[8] D. G. Coblas, Aurelian Fatu, Abdelghani Maoui, and Mohamed Hajjam: Proc IMechE Part J: J. Engineering Tribology, 229, (2015) 3.

[9] J. A. Aguilera, C. Aragon and F. Penalba: Appl. Surf. Sci., 127, (1998) 309.

[10]Zhao, Xin, and Yung C. Shin: Appl. Surf. Sci., 283, (2013) 94.

[11]Rao, B. Tirumala, Rakesh Kaul, Pragya Tiwari, and A. K. Nath: Opt. Lasers Eng., 43, (2005) 1330.

[12]A. K. Nath, D. Hansdah, S. Roy, and A. Roy Choudhury: J. Appl. Phys., 107, (2010) 123103.

[13]A. Bogaerts, Z. Chen, R. Gijbels, and A. Vertes, Spectrochimica Acta Part B, 58, (2003) 1867.

[14]J. Lu, R. Q. Xu, X. Chen, Z. H. Shen, X. W. Ni, S. Y. Zhang, and C. M. Gao: J. Appl. Phys., 95, (2004) 3890.

(Received: May 23, 2015, Accepted: April 8, 2016) 